\title{
ATPG is required for the accumulation and function of chloroplast ATP synthase in Arabidopsis
}

\author{
KONG MengMeng ${ }^{1}$, WANG FenFei ${ }^{1}$, YANG $_{\text {ZhongNan }}^{2}$ \& MI HuaLing ${ }^{1 *}$ \\ ${ }^{1}$ National Key Laboratory of Plant Molecular Genetics, Institute of Plant Physiology and Ecology, Shanghai Institutes for Biological Sciences, \\ Chinese Academy of Sciences, Shanghai 200032, China; \\ ${ }^{2}$ College of Life and Environmental Sciences, Shanghai Normal University, Shanghai 200234, China
}

Received February 28, 2013; accepted March 22, 2013; published online June 17, 2013

\begin{abstract}
The subunit II of chloroplast ATP synthase is one of the two peripheral stalks, which associates the catalytic $\mathrm{CF}_{1}$ with membrane-spanning $\mathrm{CF}_{\mathrm{o}}$. Although the structural and functional roles of chloroplast ATP synthase have been extensively examined, the physiological significance of subunit II in vivo is still unclear. In this work, we identified one Arabidopsis T-DNA insertion mutant of atpG gene encoding the subunit II of chloroplast ATP synthase. The atpg null mutant displayed an albino lethal phenotype, as it could not grow photoautotrophically. Transmission electron microscopy analysis showed that chloroplasts of atpg lacked the organized thylakoid membranes. Loss of subunit II affected the accumulation of $\mathrm{CF}_{1}-\mathrm{CF}_{\mathrm{o}}$ complex, however, it did not seem to have an effect on the $\mathrm{CF}_{1}$ assembly. The light induced ATP formation of atpg was significantly reduced compared with the wild type. Based on these results, we suggested that ATPG was essential for the accumulation and function of chloroplast ATP synthase.
\end{abstract}

ATPG, subunit II of chloroplast ATP synthase, albino, Arabidopsis

Citation: Kong M M, Wang F F, Yang Z N, et al. ATPG is required for the accumulation and function of chloroplast ATP synthase in Arabidopsis. Chin Sci Bull, 2013, 58: 3224-3232, doi: 10.1007/s11434-013-5916-x

The chloroplast ATP synthase $\left(\mathrm{CF}_{1}-\mathrm{CF}_{\mathrm{o}}\right.$ complex, $\mathrm{H}^{+}-$ ATPase) is a multisubunit complex that catalyzes ATP synthesis from ADP and orthophosphate coupling with the proton electrochemical potential across thylakoid membranes. It is mainly located in the unstacked regions of thylakoid membranes. The chloroplast ATP synthase consists of two parts, the hydrophilic $\mathrm{CF}_{1}$ (subunit $\alpha_{3}, \beta_{3}, \gamma, \delta$ and $\varepsilon$ ) and the membrane integrated $\mathrm{CF}_{\mathrm{o}}$ (subunits I, II, III 14 and IV). The $\mathrm{CF}_{1}$ contains the nucleotide binding, catalytic and regulatory sites of ATP complex. The $\mathrm{CF}_{\mathrm{o}}$ is involved in the proton transport through thylakoids [1]. These two parts are connected by a central stalk consisting of subunits $\gamma$, $\varepsilon$, III $_{14}$ and a peripheral stalk composed of subunits I, II and $\delta$ [2]. The proton movement through subunit III complex drives the rotation of subunits $\gamma$ and $\varepsilon$ in catalytic core of $\alpha_{3} \beta_{3}$ hexamer to catalyze ATP synthesis [3,4]. Many

*Corresponding author (email: mihl@sippe.ac.cn) researches have been carried out to explore the construction and function of chloroplast ATP synthase by biochemical technologies in vitro [5,6].

The peripheral stalk, which locates at the edge of chloroplast ATP synthase, is described as a highly flexible and elastic structure to stabilize the rotating machinery $[7,8]$. Two stalks were observed directly by electron microscopy of negatively stained $\mathrm{CF}_{1}-\mathrm{CF}_{\mathrm{o}}$ particles in 1998 [9]. Different species exhibit different subunit compositions of the peripheral stalk. In E. coli, it is composed of two copies of subunit $\mathrm{b}$ to form a homodimer, which has the left-handed coiled-coil interactions [10]. However, $b^{\prime} b$ heterodimer in photosynthetic bacteria constitutes the peripheral stalk corresponding to subunits II and I of chloroplast ATP synthase [11]. Different subunit compositions of the peripheral stalk in photosynthetic organisms appear to be a better adaptation to combine with different sites of $\mathrm{CF}_{1}$ [12]. Although the construction and function of subunit $\mathrm{b}$ in $E$. coli have been 
studied in great detail $[13,14]$, the knowledge about subunits II and I of chloroplast ATP synthase is limited. The nuclear atp $G$ gene encoding the subunit II of chloroplast ATP synthase has been thought to be a duplication of atpF encoding the subunit I [15], but the secondary structures of two subunits are different significantly. In Spinach, the subunit II is shorter than subunit I and it has been predicted to contain less $\alpha$-helix [12]. The subunit II has a membrane-anchored part at $\mathrm{N}$-terminus and a hydrophilic part at $\mathrm{C}$-terminus interacting with $\mathrm{CF}_{1}$ subunits [16].

The chloroplast ATP synthase contains nine subunits and six of them are encoded by the chloroplast genes locating in two gene clusters [17]. Only subunits, $\gamma, \delta$ and II are encoded by the nuclear genes $a t p C$, atpD and atp $G$, respectively. Two Arabidopsis seedling-lethal mutants (dpal and atpd) for the genes encoding $\gamma$ and $\delta$ subunits of ATP synthase have already been characterized $[18,19]$. The subunit $\gamma$ contains the redox regulatory domain of ATP synthase [20]. Recently, evidence showed that subunit $\gamma$ catalyzes ATP hydrolysis-driven proton translocation in non-photosynthetic plastids, suggesting its roles in maintaining sufficient transthylakoid proton gradient to drive protein translocation or other processes [21]. Deficiency of subunits, $\gamma$ (ATPC) or $\delta$ (ATPD) affects the composition and function of chloroplast ATP synthase. As a consequence of destabilized ATP complex, accumulation of increased proton gradient through the thylakoid membranes induces high non-photochemical quenching in both mutants. However, the genetic and physiological studies on ATPG are still lacking. In this study, we have identified the atpg mutant from Arabidopsis T-DNA insertion lines. We investigated the effect of ATPG on the function and constitution of chloroplast ATP synthase. We also discuss the possible role of ATPG in chloroplast development.

\section{Materials and methods}

\subsection{Plant materials and growth conditions}

The Arabidopsis wild-type plants used in this study were Columbia-0 (Col). The atpg mutants were screened from pSKI15 activation-tagging T-DNA mutant pools [22]. Surface-sterilized seeds were sown on Murashige and Skoog (MS) medium supplemented with $2 \%$ sucrose at $22^{\circ} \mathrm{C}$ under $12 \mathrm{~h}$ light $/ 12 \mathrm{~h}$ dark conditions at $15 \mu \mathrm{mol}$ photons $\mathrm{m}^{-2} \mathrm{~s}^{-1}$.

\subsection{Molecular cloning of $\operatorname{atp} G$ gene and complementation}

The Tail-PCR and arbitrary degenerate primers used in this work were as described [23]. T-DNA insertion site was identified by PCR amplified with primers of T-DNA left border (AtLB1, 5'-ATACGACGGATCGTAATTTGTC-3'; AtLB2, 5'-TAATAACGCTGCGGACATCTAC-3'; AtLB3 5'-TTGACCATCATACTCATTGCTG-3') and gene specific primers (LP, 5'-CGACAAGGTCTATTACTCTCCG-3';
RP, 5'-CTGGAGTGTTTGGCTCTGAA-3'). For mutants, PCR with AtLB3 and RP primers amplified a DNA fragment about $450 \mathrm{bp}$. For wild-type plants, PCR with LP and RP primers amplified a DNA fragment about 780 bp. For heterozygous plants, PCR showed both fragments. For complementation analyses, full-length cDNA fragment of atp $G$ was cloned into binary vector pMON530 under the control of CaMV 35S promoter. Specific primers 5'-ATGGCTGCTAATTCGATAATG-3' and 5'-GACGAACAATCCAAGAAACAG-3' were chosen for PCR amplification. A high-fidelity phusion DNA polymerase (BioLabs) was used and the construct was verified by sequencing. Complementation construct was transformed into heterozygous plants using floral dip transformation as described [24]. The transformants were selected on MS medium with $40 \mathrm{mg} \mathrm{L}^{-1}$ kanamysin.

\subsection{Chlorophyll analysis}

Total chlorophylls were determined according to Lichtenthaler [25]. Fresh leaves from two-week-old seedlings were homogenized in $80 \%$ acetone for $12 \mathrm{~h}$ at $4^{\circ} \mathrm{C}$. Spectrophotometric quantification was carried out in a HITACHI U-1900 spectrophotometer. Pigment measurements were repeated in three independent experiments.

\subsection{Transmission electron microscopy}

Leaf segments were fixed with $2.5 \%$ glutaraldehyde in phosphate buffer ( $\mathrm{pH} 7.2$ ) for $24 \mathrm{~h}$ at $4^{\circ} \mathrm{C}$ and then washed three times with the same buffer. The tissues were postfixed overnight in $1 \% \mathrm{OsO}_{4}$ at $4^{\circ} \mathrm{C}$. The fixed samples were dehydrated through a series of ethanol solutions, infiltrated with a series of epoxy resin in epoxy propane and embedded in Epon 812 resin. Ultrathin sections were cut with a diamond knife and mounted onto copper grids. Then the samples were stained with $2 \%$ uranyl acetate for $10 \mathrm{~min}$ followed by lead citrate for 2 min and observed with a transmission electron microscope (Phillips CM120).

\subsection{RT-PCR and northern analysis}

Total RNA was isolated from three-week-old plants with RNAgents Denaturing Solution (Promega). RNA samples $(2-5 \mu \mathrm{g})$ were used as templates for cDNA synthesis using SuperScript III First-Strand synthesis system (Invitrogen) according to the manufacturer's instructions. RT-PCR reactions were performed with specific primers for at $4 \mathrm{~g} 32260$ (5'-CTAATCTCCCTGTCATCCAACC-3' and 5'-GAGCAGCAATCTGAGAATCCA-3') its neighbor genes at $4 g 32250$ (5'-CAAAGAAGGGTTGTGGTAGA-3' and 5'-TCAGAACTTGGTAAAGGGATA-3'), and $a t 4 g 32270$ (5'-TTTCTCACTTGCCCATCTC-3' and 5'-CATCCTTCTCGGAATTACAC-3'). Actin2 (at3g18780) was used as an internal control with its primers (5'-TCTTCTTCCGCTCTTTCTTTCC-3' 
and 5'-TCTTACAATTTCCCGCTCTGC-3'). Northern analysis was performed as described [26]. Primer pairs are listed in Table S2.

\subsection{Preparation of thylakoids}

Fresh leaves of three-week-old plants were grinded with icecold STN solution containing $50 \mathrm{mmol} \mathrm{L}^{-1}$ Tris- $\mathrm{HCl}(\mathrm{pH} 7.4)$, $400 \mathrm{mmol} \mathrm{L}^{-1}$ sucrose, $10 \mathrm{mmol} \mathrm{L}^{-1} \mathrm{NaCl}$ and $2 \mathrm{mmol} \mathrm{L}^{-1}$ $\mathrm{MgCl}_{2}$. The extract was filtered through a nylon membrane and centrifuged at $200 \times g$ for $3 \mathrm{~min}$ at $4{ }^{\circ} \mathrm{C}$. The supernatant was centrifuged at $6000 \times g$ for $10 \mathrm{~min}$, washed twice with STN solution and resuspended in STN solution.

\subsection{Immunoblot analysis}

Thylakoid membrane proteins containing $2 \mu \mathrm{g}$ of chlorophyll were denatured with SDS sample buffer in a boiling water bath for $5 \mathrm{~min}$ and separated by SDS-PAGE in a 12\% polyacrylamide gel with $4 \mathrm{~mol} \mathrm{~L}^{-1}$ urea. Then the proteins were transferred electrophoretically onto a PVDF membrane (Millipore) and incubated with different antibodies against chloroplast proteins (Agrisera). Signals were identified by an ECL plus Western blotting detection system (GE) as manufacturer's instructions.

\subsection{Two-dimensional blue native/SDS-PAGE}

One-dimensional blue native-polyacrylamide gel electrophoresis (BN-PAGE) was carried out as described [27,28]. Thylakoid membrane proteins isolated from three-week-old plants were solubilized in $25 \mathrm{mmol} \mathrm{L}{ }^{-1}$ BisTris- $\mathrm{HCl}(\mathrm{pH}$ 7.0 ), $20 \%$ glycerol and $2 \%$ dodecyl- $\beta$-D-maltopyranoside (DM). After incubation on ice for $15 \mathrm{~min}$, samples were centrifuged at $16000 \times g$ for $20 \mathrm{~min}$ at $4^{\circ} \mathrm{C}$. The supernatant was thoroughly mixed with loading buffer $(10: 1$, v/v) containing $100 \mathrm{mmol} \mathrm{L}^{-1}$ BisTris- $\mathrm{HCl}\left(\mathrm{pH} \mathrm{7.0),} 500 \mathrm{mmol} \mathrm{L}^{-1}\right.$ 6-aminocaproic acid, 30\% sucrose and 5\% Coomassie Blue G250. Then each sample equivalent to $5 \mu \mathrm{g}$ of chlorophyll was loaded on a $4.5 \%-12 \%$ polyacrylamide gradient gel. For two-dimensional gel analysis, the gel strips obtained by BN-PAGE were stained with Coomassie brilliant blue and incubated in SDS sample buffer containing 5\% $\beta$-mercaptoethanol for $15 \mathrm{~min}$. Then gel strips were put on the top of SDS-PAGE in a $12 \%$ polyacrylamide gel with $4 \mathrm{~mol} \mathrm{~L}^{-1}$ urea. After electrophoresis, the proteins were transferred onto a PVDF membrane for immunoblot analysis.

\subsection{Measurement of light induced ATP production}

The prepared thylakoids were added to $1 \mathrm{~mL}$ reaction solution containing $50 \mathrm{mmol} \mathrm{L}^{-1}$ Tris- $\mathrm{HCl}\left(\mathrm{pH}\right.$ 7.6), $5 \mathrm{mmol} \mathrm{L}{ }^{-1}$ $\mathrm{NaCl}, 5 \mathrm{mmol} \mathrm{L} \mathrm{MgCl}_{2}, 10 \mathrm{mmol} \mathrm{L}{ }^{-1} \mathrm{Na}_{2} \mathrm{HPO}_{4}, 1 \mathrm{mmol} \mathrm{L}{ }^{-1}$ FeCY and $1 \mathrm{mmol} \mathrm{L}^{-1} \mathrm{ADP}$. The reaction mixture was in- cubated for $1 \mathrm{~min}$ under illumination of $85 \mu \mathrm{mol}$ photons $\mathrm{m}^{-2} \mathrm{~s}^{-1}$ at $25^{\circ} \mathrm{C}$ and inactivated by boiling water bath for 5 min. The ATP synthesis was measured by a luciferase luminescence assay [29].

\subsection{Accession numbers}

Sequence data from this article can be found in the GenBank data libraries under accession number: Volvox carteri (AAD55575), Chlamydomonas reinhardtii (EDP00272), Oryza sativa (NP_001049730), Sorghum bicolor (EER92459), Spinacia (CAA50520), Vitis vinifera (XP_002285183), Populus trichocarpa (ABK94675), Physcomitrella patens (XP_001754735.1), Cyanophora paradoxa (AAA81256) and Arabidopsis ATPG (AT4G32260).

\section{Results}

\subsection{The nuclear atp $G$ gene encodes the subunit II of chloroplast ATP synthase}

The subunit II of chloroplast ATP synthase (ATPG) is a polypeptide of 219 amino acids with a molecular weight of $23 \mathrm{kD}$. Structural analysis revealed that ATPG contains a highly conserved domain belonging to ATP-synt_B family at C-terminal. The TargerP [30] and Predotar [31] programs predicted that ATPG protein is targeted to chloroplast. Sequence alignment showed that the ATPG homologs existed in various plants, including Spinacia [15], Vitis vinifera, Oryza sativa, Populus trichocarpa, Sorghum bicolor and Physcomitrella patens. In addition, the homologs were also found in Volvox carteri, Chlamydomonas reinhardtii and Cyanophora paradoxa (Figure 1(a)). We further performed a phylogenetic analysis of ATPG and its homologs to investigate the evolutionary relationship among these proteins. As shown in Figure 1(b), the proteins from angiosperms formed one clade. The proteins from monocots and dicots were divided into two separated groups within the angiosperm clade. While the proteins from alga were distant from angiosperms and bryophyte. Our data indicated that the monocot-dicot divergence was well reflected from the phylogenetic tree. Therefore, ATPG is a highly conserved ancient protein, which exists widely in photosynthetic organisms.

To analyze the atp $G$ expression profile, we isolated total RNA from various tissues of three-week-old wild type. RT-PCR analysis showed that atp $G$ mRNA was detectable in all tissues with high expression in rosette and cauline leaves, but it was weakly expressed in roots and siliques (Figure 1(d)). As an important signal, light plays a vital role during the process of proplastids differentiating into chloroplasts. Many genes involved in photosynthsis are expressed after exposure to 6-24 h illumination [33,34]. To examine the effect of light on atpG mRNA expression, we extracted total RNA from five-day-old etiolated seedlings exposed to $0,2,6,9,12$ and 24 h of continuous light, respectively. RT- 
(a)

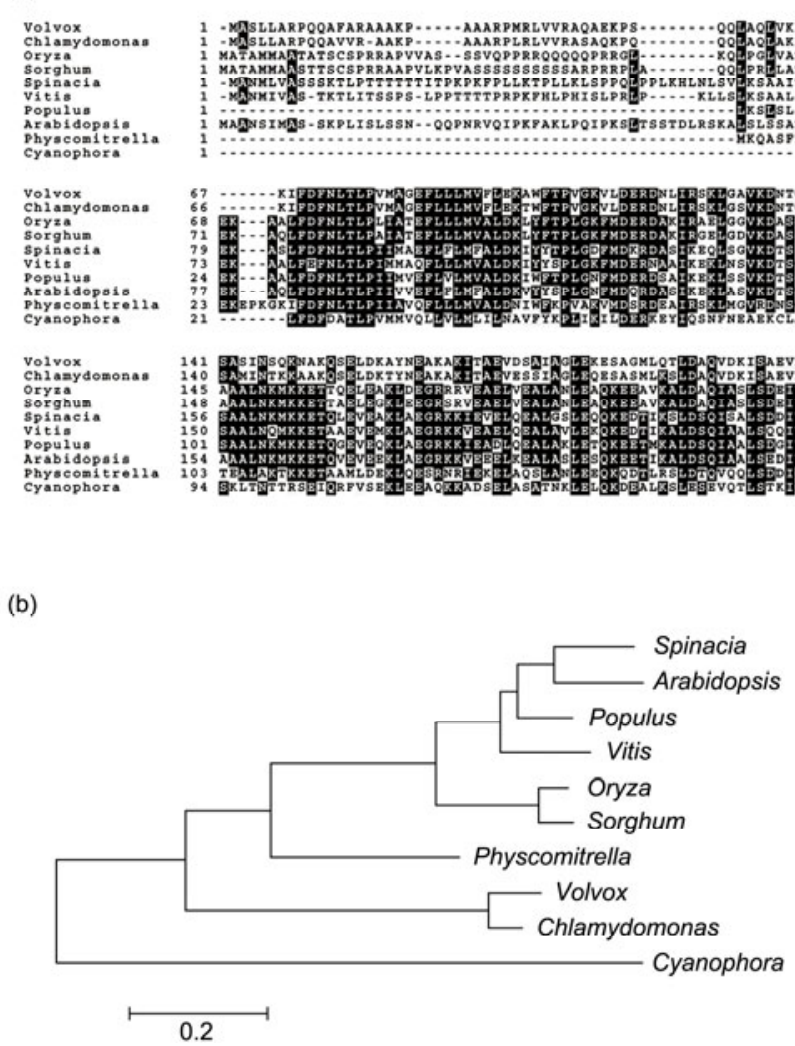

(c)

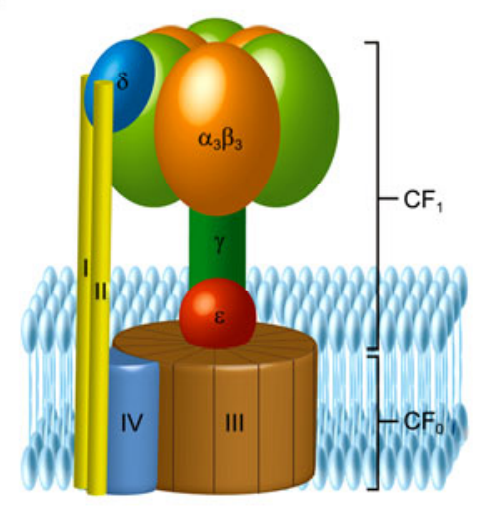

(d)

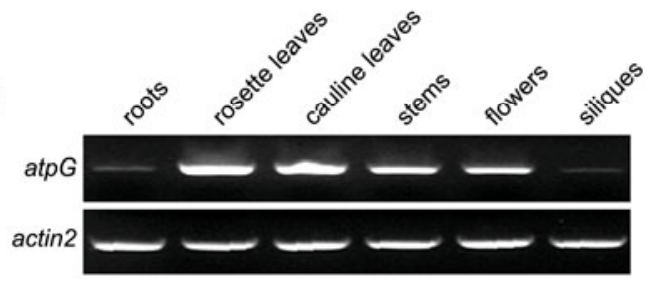

(e)

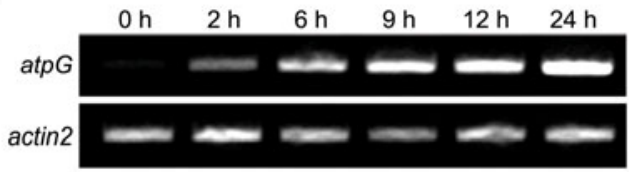

Figure 1 Structural organization of chloroplast ATP synthase and sequence analysis of the ATPG protein. (a) Alignment analysis between ATPG and its homologs. The sequences were aligned using Clustal W [32] and displayed using BOXSHADE (www.ch.embnet.org/software/BOX_form.html); (b) unrooted phylogenetic tree of ATPG and its homologs. Amino acid sequences were analyzed by MEGA 5.0; (c) the structure of chloroplast ATP synthase is based on the model of Richter et al. [4]; (d) tissue-specific expression of atpG gene. Total RNA was extracted from various tissues of wild-type plants; (e) light induction of atp $G$ gene. Total RNA was extracted from five-day-old etiolated wild-type seedlings treated with illumination for $0,2,6,9,12$ and $24 \mathrm{~h}$, actin 2 gene was used as an internal control.

PCR results showed that atp $G$ was slightly expressed in the dark, but after transfer of the etiolated plants to light, atpG mRNA was gradually increased (Figure 1(e)). This result suggests that the expression of atp $G$ mRNA is induced by light.

\subsection{Knockout of atpG leads to the seedling-lethal phenotype}

We screened the atpg mutant from Arabidopsis pSKI15 activation-tagging T-DNA mutant pools [22]. The mutant was albino and seedling lethal on soil. When cultivated on sucrose-supplemented medium, the mutant survived for three weeks with 6-8 leaves (Figure 2(a)). Thermal asymmetric interlaced (TAIL) PCR analysis revealed that the T-DNA was inserted into the second exon of atp $G, 649 \mathrm{bp}$ downstream from the ATG start codon (Figure 2(b)). No chlorophyll fluorescence signal was detected in the mutant, which might have been a result of low chlorophyll content of atpg (Table S1). The microscopy analysis showed that the mutant had less mesophyll cells, which were loosely arranged around the vascular with larger intercellular spaces (Figure S1).
When we backcrossed the heterozygous plants with wild type, all the $\mathrm{F}_{1}$ plants behaved like wild type. $\mathrm{F}_{2}$ progenies segregated at a $3: 1$ ratio (green: albino $=537: 169 ; \chi^{2}=0.37$, $P<0.05$ ), indicating that atp $g$ was caused by a single recessive mutation. RT-PCR results showed that no expression of atp $G$ was detected in mutant, whereas the transcripts of its neighbor genes were at similar levels as those in wild type (Figure 2(c)). In accordance with RT-PCR results, ATPG protein was undetectable in the mutant by immunodetection (Figure 2(d)). To further confirm the albino phenotype was caused by a T-DNA insertion into atp $G$ locus, we carried out the complementation experiment. Full-length atp $G$ cDNA under the control of CaMV 35S promoter was transformed into heterozygous plants. We obtained 16 independent transformants with the mutant background, and all these lines had restored the wild-type phenotype (Figure S2). These results demonstrated that the mutation was due to a lack of atp $G$ function.

Since atpg mutant exhibited an albino phenotype, we further compared the ultrastructure of chloroplasts in both ecotypes grown under different light conditions by transmission electron microscopy. As shown in Figure 2(e), the wild-type chloroplasts contained well-structured thylakoid 
(a)

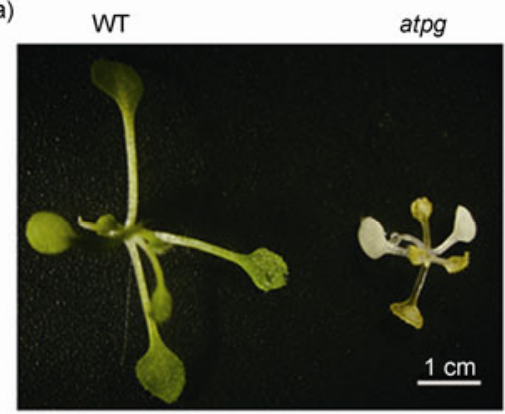

(b)

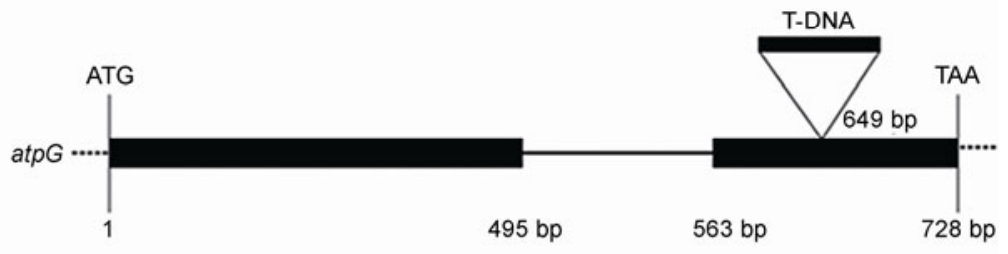

(e) $15 \mu \mathrm{mol}$ photons $\mathrm{m}^{-2} \mathrm{~s}^{-1}$ 80 umol photons $\mathrm{m}^{-2} \mathrm{~s}^{-1}$

(c)

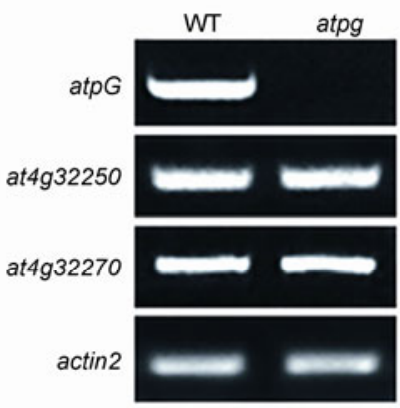

(d)

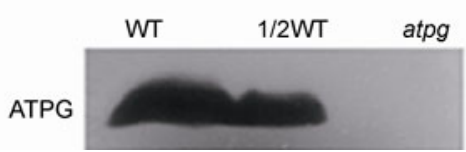

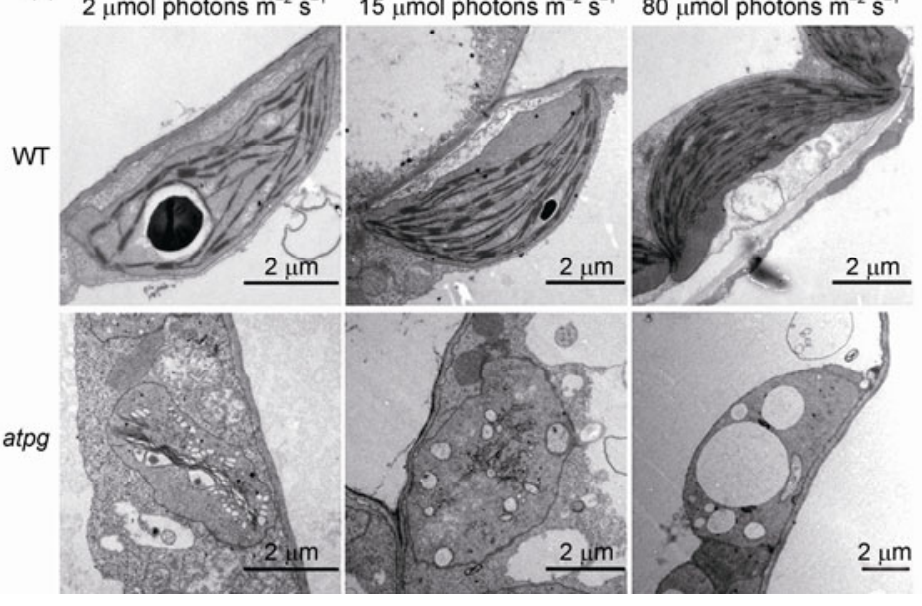

Figure 2 Visible phenotype of wild type (WT) and atpg. (a) Photograph of seedlings growth on MS medium supplemented with $2 \%$ sucrose at $15 \mu$ mol photons $\mathrm{m}^{-2} \mathrm{~s}^{-1}$; (b) schematic representation of the T-DNA insertion into atp $G$ gene. The boxes and bar represent exons and intron, respectively. The numbers below show the position of exon and T-DNA insertion; (c) RT-PCR analysis of atpG and its neighbor genes; (d) immunoblot analysis of ATPG protein; (e) transmission electron microscope of chloroplasts from wild type and atpg. The second leaves of two-week-old plants grown under different light intensity were fixed for transmission electron microscope analysis, scale bars are indicated.

membranes composed of stroma thylakoids and granna thylakoids. While in the atpg mutant, some stacked but disorganized thylakoid membranes with swollen lumen space were observed when grown under low intensity light of $2 \mu \mathrm{mol}$ photons $\mathrm{m}^{-2} \mathrm{~s}^{-1}$. When light intensity increased to $15 \mu \mathrm{mol}$ photons $\mathrm{m}^{-2} \mathrm{~s}^{-1}$, only few short appressed membranes and vesicle-like structures were observed. The chloroplasts were completely vacuolated lacking the internal membrane when grown under normal light of $80 \mu \mathrm{mol}$ photons $\mathrm{m}^{-2} \mathrm{~s}^{-1}$. These results indicated that the chloroplast development in atpg was arrested at an early stage, especially during thylakoid membrane formation and this might have been aggravated by photo-oxidative stress.

\subsection{The impaired accumulation of other subunits of ATP synthase in atpg}

To investigate the level of other subunits of ATP synthase in the absence of subunit II, chloroplast proteins from threeweek-old wild type and atpg leaves were isolated for immunoblot analysis. We examined all five subunits of $\mathrm{CF}_{1}$ and detected four of them (subunits $\alpha, \beta, \gamma$ and $\varepsilon$ ) in atpg.
The amount of subunit $\gamma$ was significantly reduced compared to the wild type. However, the level of subunit $\varepsilon$ was increased, which might be due to the fact that the protein concentration in atpg was more than ten times of those in the wild type, when equal amounts of chlorophyll were loaded. No accumulation of subunit I was detected in atpg, indicating that the mutation probably caused the destabilization of membrane integrated $\mathrm{CF}_{\mathrm{o}}$ (Figure 3(a)). We further examined the transcript levels of genes encoding ATP synthase subunits. Northern analysis demonstrated that the plastid-encoded genes atpA, atpB, atpF and nuclear-encoded genes atp $C$ and $a t p D$ did not show major changes in atpg (Figure 3(c)). This result suggested that a severe defect in the accumulation of other subunits of ATP synthase in atpg was not due to the defective transcriptional activity.

In addition, we also checked the changes of other photosynthetic proteins in atpg. Immunoblot analysis showed that the photosystem II reaction center D1 (PsbA) and D2 (PsbD) proteins, cytochrome $f$ (Cytf) were undetected in atpg. Levels of CP47 protein of photosystem II (PsbB), the D subunit of photosystem I (PsaD), the chlorophyll-binding protein Lhca1 and Lhcb1 were slightly decreased compared to thoses 

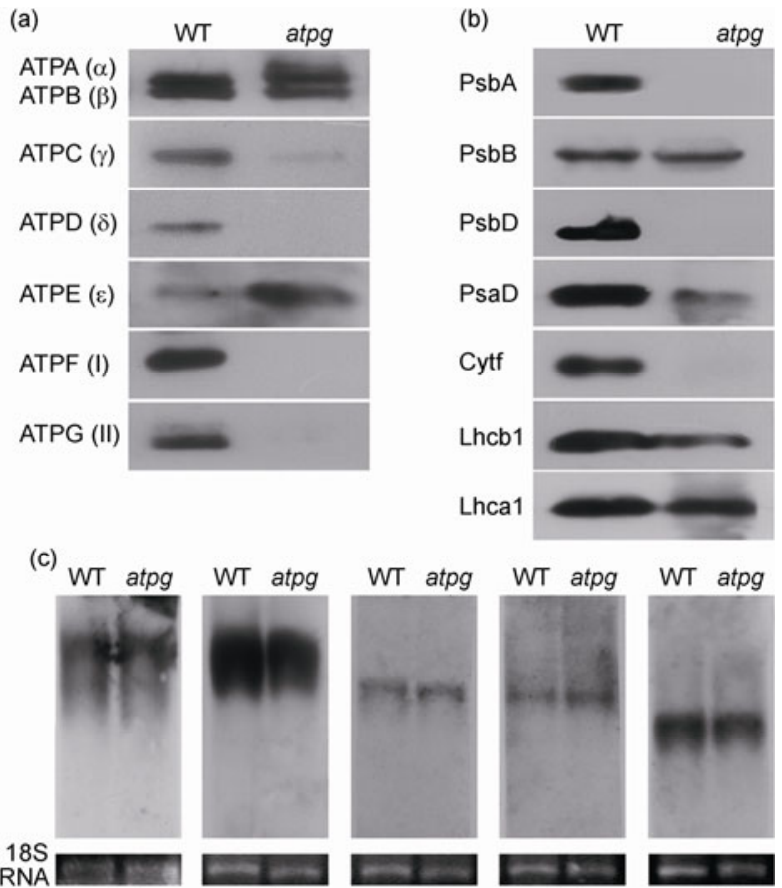

$\operatorname{atp} A$

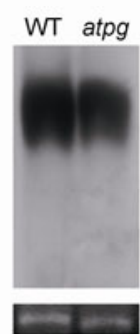

atpB

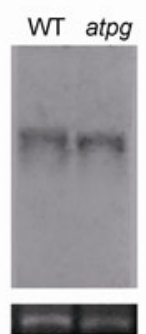

$\operatorname{atpC}$
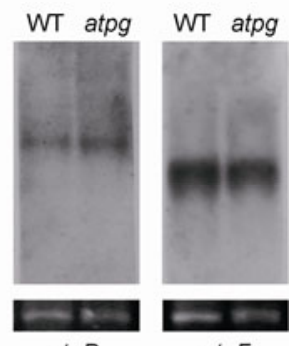

$\operatorname{atp} D$

$\operatorname{atp} F$

Figure 3 Immunoblot analysis of chloroplast proteins and northern analysis of nuclear- and plastid-encoded genes in wild type and atpg. (a) Immunoblot analysis of chloroplast ATP synthase subunits; (b) immunoblot analysis of other chloroplast proteins. Thylakoid membranes containing $2 \mu \mathrm{g}$ of chlorophyll were separated by SDS-PAGE, transferred to a PVDF membrane and immunoblotted with antibodies against photosynthetic proteins; (c) Northern analysis of nuclear- and plastid-encoded genes. About $10 \mu \mathrm{g}$ total RNA was transferred to a nylonmembrane after electrophoresis and further probed with DIG-labeled probes for atpA, atpB, atpC, atpD and atpF.

in the wild type (Figure 3(b)).

\subsection{The defect in the $\mathrm{CF}_{1}-\mathrm{CF}_{0}$ complex in atpg}

The subunit II together with subunit I act as a peripheral stalk to connect the $\mathrm{CF}_{1}$ and $\mathrm{CF}_{\mathrm{o}}$ [2]. To explore the function of ATPG on the enzyme accumulation, chloroplast proteins were separated by two-dimensional blue native/ SDS-PAGE and subsequently immunoblotted with specific antibodies raised against subunits $\beta$ (ATPB) and $\gamma$ (ATPC). In wild-type plants, the chloroplast ATP synthase can be separated into three distinguishable subcomplexes as previously reported [35]. The first protein spot corresponding to a molecular mass of $550 \mathrm{kD}$ represents the monomeric form of $\mathrm{CF}_{1}-\mathrm{CF}_{\mathrm{o}}$ complex, $440 \mathrm{kD}$ protein spot represents the assembled $\mathrm{CF}_{1}$ particles and $140 \mathrm{kD}$ protein spot represents free $\alpha$ and $\beta$ subunits (Figure 4). In atpg mutant, only the second and third spots were detected when probed with an antibody against subunit $\beta$, whereas the $550 \mathrm{kD}$ protein spot was missed. Moreover, the level of free subunit $\beta$ was shown to be increased relative to the wild type. The subunit $\gamma$ was also found in the assembled $\mathrm{CF}_{1}$ spot corresponding to a molecular mass of $440 \mathrm{kD}$ in atpg. These results suggested

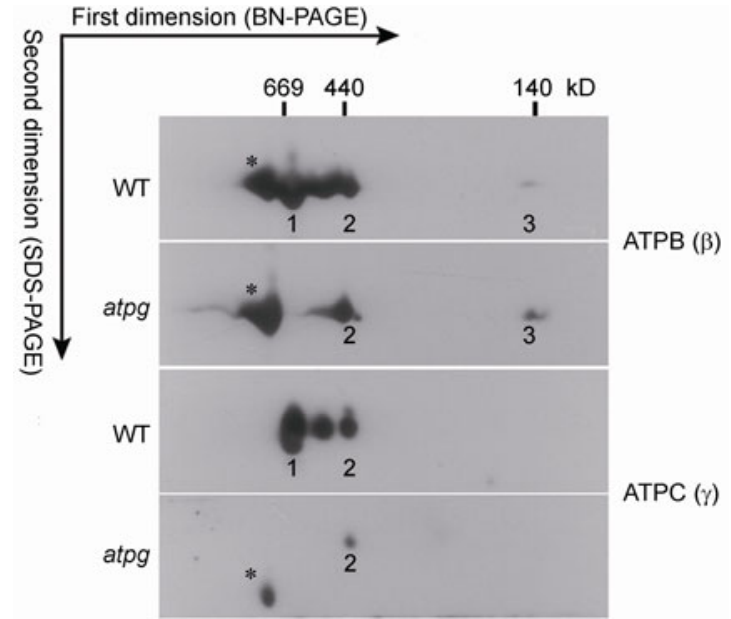

Figure 4 Immunodetection analysis of chloroplast ATP synthase subunits separated by two-dimensional blue native/SDS-PAGE in wild type and atpg. Chloroplasts from three-week-old plants containing $5 \mu \mathrm{g}$ of chlorophyll were separated by one-dimensional blue native-PAGE. Then the gel strips were subjected to SDS-PAGE in the second dimension and immunoblotted with antibodies raised against subunits $\beta$ and $\gamma$. The numbers below indicate different subcomplexes of chloroplast ATP synthase. 1 represents the monomeric form of ATP complex, 2 represents the assembled $\mathrm{CF}_{1}$ part, 3 represents free $\alpha$ and $\beta$ subunits, respectively. The asterisks (*) indicate the non-specific binding of antibodies with unknown proteins.

that subunits $\beta$ and $\gamma$ were probably assembled into $\mathrm{CF}_{1}$ without the subunit II, but the accumulation of integral $\mathrm{CF}_{1}-\mathrm{CF}_{\mathrm{o}}$ complex was disturbed.

\subsection{The suppression of the activity of light induced ATP production in atpg}

Adenosine triphosphate (ATP) has long been recognized as a universal energy currency of living cells. In chloroplast, ATP is required for the photosynthetic assimilation of carbon dioxide into carbohydrates. To study whether the chloroplast ATP synthase activity is affected in the absence of subunit II, we measured the light induced ATP production in both ecotypes using potassium ferricyanide $(\mathrm{FeCY})$ as an electron acceptor. As shown in Figure 5, the content of light induced ATP synthesis in the wild type was about $39.26 \pm$ $9.45 \mu \mathrm{mol} \mathrm{\textrm {mg } ^ { - 1 }} \mathrm{chl} \mathrm{h}^{-1}$. In contrast, it was $62.8 \%$ lower in atpg than that of wild type $\left(14.61 \pm 7.23 \mu \mathrm{mol} \mathrm{mg}^{-1} \mathrm{chl} \mathrm{h}^{-1}\right)$. The result indicates that deletion of subunit II of chloroplast ATP synthesis causes the severe suppression of photophosphorylation activity.

\section{Discussion}

\subsection{ATPG is essential for the $\mathbf{C F}_{1}-\mathrm{CF}_{\mathbf{0}}$ complex accu- mulation in chloroplast}

It is well known that chloroplast ATP synthase catalyzes the light-dependent synthesis of ATP coupling with transmembrane proton transport. In higher plants, isolated chloroplasts 


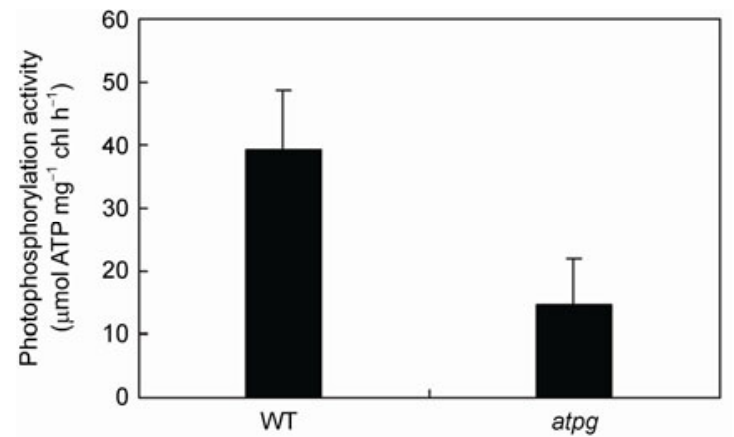

Figure 5 The light induced ATP production of chloroplasts in wild type and atpg. The reactions were carried out at room temperature and potassium ferricyanide (FeCY) was used as an electron acceptor. The ATP content was measured by a luciferase luminescence assay.

synthesize most ATP synthase subunits that are chloroplastencoded. These chloroplast-made components can form a subcomplex at the earlier stage of ATP synthase assembly [36]. Only subunits $\gamma, \delta$ and II are synthesized outside the chloroplast and then imported. Previous studies have shown that the chloroplast ATP synthase was destabilized without subunits $\gamma$ or $\delta$, suggesting that both were essential for the enzyme assembly $[18,19]$.

The subunit II of $\mathrm{CF}_{\mathrm{o}}$ functions as a peripheral stalk to link the hydrophilic $\mathrm{CF}_{1}$ with membrane-spanning $\mathrm{CF}_{\mathrm{o}}$. Complete deletion of this subunit in cyanobacterium is lethal, suggesting the subunit $b^{\prime}$ is essential for cell viability [37]. In this study, we reported the isolation and characterization of a null mutant of atpG encoding the subunit II of ATP synthase in Arabidopsis, which might shed light on the assembly mechanism of chloroplast ATP enzyme. Choquet and Vallon [38] have pointed out that the assembly of ATP synthase is an intricate process involving various inter-subunit interactions within $\mathrm{CF}_{1}$ and $\mathrm{CF}_{\mathrm{o}}$ or between these subcomplexes. Some protein factors have been proven to interact with certain ATP synthase subunits to stabilize or promote assembly of the enzyme, such as Alb4 [35]. By two-dimensional blue native/SDS-PAGE analysis, we found that lack of subunit II affected the $\mathrm{CF}_{1}-\mathrm{CF}_{\mathrm{o}}$ complex accumulation (Figure 4). This result suggests that the subunit II might be involved in the stabilization and/or assembly of $\mathrm{CF}_{1}-\mathrm{CF}_{\mathrm{o}}$ complex. However, it is not clear if this is the secondary effect of other ATP synthase subunits deficiency. Together with dpal and atpd mutants $[18,19]$, we conclude that the chloroplast ATP synthase is a highly organized complex, one subunit deletion often affects the accumulation of whole complex.

The $\mathrm{CF}_{1}-\mathrm{CF}_{\mathrm{o}}$ complex is able to catalyze a reversible reaction: ATP synthesis and hydrolysis. When treated with EDTA or other chelating agents, the pure $\mathrm{CF}_{1}$ could be isolated from thylakoid membranes. The isolated $\mathrm{CF}_{1}$ is a latent ATPase [39]. Despite the fact that atpg lacked the organized thylakoid membranes, four subunits (subunits $\alpha, \beta$, $\gamma$ and $\varepsilon$ ) appeared to assemble into an incomplete $\mathrm{CF}_{1}$ com- plex which was not associated with the thylakoid membranes. This result further proved that the subunit II is involved in $\mathrm{CF}_{1}$ integrating into the proton channel $\mathrm{CF}_{\mathrm{o}}$. Our result was consistent with some ATP synthase mutants of Chlamydomonas reinhardtii, in which the mutants defective in $\mathrm{CF}_{\mathrm{o}}$ subunits allowed the accumulation of some $\mathrm{CF}_{1}$ components, but the mutant lacking a $\mathrm{CF}_{1}$ subunit appeared to lose all the $\mathrm{CF}_{\mathrm{o}}$ subunits [40]. In summary, loss of the subunit II causes the catalytic $\mathrm{CF}_{1}$ detached from transmembrane $\mathrm{CF}_{\mathrm{o}}$, but the assembly of $\mathrm{CF}_{1}$ seems to be less affected. Furthermore, we could infer that assembly of catalytic $\mathrm{CF}_{1}$ head is independent of the membrane-bound $\mathrm{CF}_{\mathrm{o}}$.

\subsection{ATPG is required for the photophosphorylation activity}

In chloroplast, the light induced ATP production through the use of a proton gradient is referred to as photophosphorylation. There are three modes of photophosphorylation in vivo including cyclic, non-cyclic and pseudocyclic photophosphorylation. Cyclic photophosphorylation involves photosystem I and synthesizes ATP only. Non-cyclic photophosphorylation produces both NADPH and ATP, which could be used in the Calvin cycle [41]. In our study, the light induced ATP production of atpg was decreased to $37.2 \%$ of the wild type level (Figure 5), indicating that inactivation of subunit II inhibited the photophosphorylation activity significantly.

ATP synthesis involves the transfer of electrons from the intermembrane space, through inner membrane, back to the matrix. We noticed that although the electron transfer chain was damaged due to the photosystems deficiency in the mutant, a small amount of light induced ATP formation was still detected when substrates were supplied to the reaction. The reasonable explanation is that extensive early studies have confirmed that the main catalytic site of ATP synthase is subunit $\beta$ with some contributions from side chains in the subunit $\alpha[42,43]$. So we hypothesized that the incomplete $\mathrm{CF}_{1}$ head composing of subunits $\beta$ and $\alpha$ in the mutant maintained partial photophosphorylation activity in vivo. And the residual thylakoid membranes in atpg might provide a prerequisite for the catalysis of ATP formation.

\subsection{ATPG is required for the chloroplast development}

Mutations of many nuclear genes that are involved in chloroplast development would cause albino phenotype. The chloroplasts of these mutants often lack internal membrane or contain few appressed membranes, such as apg3, $d x r$ and agyl [44-46]. Electron microscopy analysis showed that the chloroplast development in atpg was blocked even grown under low light conditions (Figure 2(e)). These findings suggest that ATPG is required for chloroplast development, especially during the thylakoid formation. In higher plants, chloroplast development is an extremely complex procedure, 
which involves many energy-dependent processes. Generally, at the onset of illumination, Pchlide is converted to Chlide and the prolamellar body breaks up into some small vesicles, the photosystem I and II reaction centers start to form in these vesicles and the photosystem I-mediated cyclic photophosphorylation appears within the first few minutes $[47,48]$. Baker and Butler [49] inferred that these vesicles contain an active proton pump, implying that photophosphorylation has an important role at the early stage of chloroplast development. In this study, the loss of the subunit II affected the $\mathrm{CF}_{1}-\mathrm{CF}_{\mathrm{o}}$ complex accumulation, further impaired the photophosphorylation activity. Therefore, it is reasonable to assume that the defective chloroplast development in atpg results from the lack of ATP supply. As previously reported, knockout of subunit $\gamma$ affected the chloroplast development [18], we conclude that inactivation of single subunit of chloroplast ATP synthase would lead to the blockage of chloroplast development.

On the other hand, chloroplast ATP synthase is one of the four photosynthetic complexes localized in thylakoid membranes. Failure in the assembly of ATP synthase would also affect the elongation of thylakoids and the secondary effect of impaired thylakoids is degradation of most thylakoid proteins. As shown in Figure 3(b), some core proteins of photosystem were absent in atpg, including $\mathrm{CF}_{\mathrm{o}}$ subunits. Incomplete photosystems would cause the photo-damage of photosynthetic organization so that the mutant could hardly survive even under weak light conditions.

\section{Conclusions}

In this study, we isolated one Arabidopsis T-DNA insertion mutant of atp $G$ gene encoding the subunit II of chloroplast ATP synthase. We demonstrated that loss of subunit II affects the accumulation of $\mathrm{CF}_{1}-\mathrm{CF}_{\mathrm{o}}$ complex, resulting in impairment of the function of chloroplast ATP synthase and chloroplast development. We suggest that the subunit II is of structural and functional importance for the chloroplast ATP synthase.

We sincerely thank Dr. QU LiJia from Peking University for providing the Arabidopsis T-DNA insertion lines. We are grateful to our colleagues $M r$. GU JianBen for skillful technical assistance with photophosphorylation measurement, Prof. SHEN YunKang, WEI JiaMian and CHEN GenYun for the fruitful discussion of this work. We also thank Dr. YU QingBo from Shanghai Normal University for thoughtful comments of the manuscript. This work was supported by the National Natural Science Foundation of China (31070215 and 31100181) and the State Key Basic Research and Development Program of China (2009CB118504).

1 Fromme P, Boekema E J, Gräber P. Isolation and characterization of a supramolecular complex of subunit III of the ATP-synthase from chloroplasts. Z Naturforsch, 1987, 42: 1239-1245

2 Poetsch A, Rexroth S, Heberle J, et al. Characterisation of subunit III and its oligomer from spinach chloroplast ATP synthase. Biochim Biophys Acta, 2003, 1618: 59-66
3 McCarty R E, Evron Y, Johnson E A. The chloroplast ATP synthase: A rotary enzyme? Annu Rev Plant Physiol Plant Mol Biol, 2000, 51: 83-109

4 Richter M L, Samra H S, He F, et al. Coupling proton movement to ATP synthesis in the chloroplast ATP synthase. J Bioenerg Biomembr, 2005, 37: 467-473

5 Feng Y, McCarty R E. Subunit interactions within the chloroplast ATP synthase $\left(\mathrm{CF}_{0}-\mathrm{CF}_{1}\right)$ as deduced by specific depletion of $\mathrm{CF}_{\mathrm{o}}$ polypeptides. J Biol Chem, 1990, 265: 12481-12485

6 Vollmar M, Schlieper D, Winn M, et al. Structure of the c14 rotor ring of the proton translocating chloroplast ATP synthase. J Biol Chem, 2009, 284: 18228-18235

7 Fillingame R H, Jiang W, Dmitriev O Y. Coupling $\mathrm{H}^{+}$transport to rotary catalysis in F-type ATP synthases: Structure and organization of the transmembrane rotary motor. J Exp Biol, 2000, 203: 9-17

8 Junge W, Pänke O, Cherepanov D A, et al. Inter-subunit rotation and elastic power transmission in $\mathrm{F}_{0} \mathrm{~F}_{1}$-ATPase. FEBS Lett, 2001, 504: $152-160$

9 Böttcher B, Schwarz L, Gräber P. Direct indication for the existence of a double stalk in $\mathrm{CF}_{0} \mathrm{~F}_{1}$. J Mol Biol, 1998, 281: 757-762

10 Wise $\mathrm{J}$ G, Vogel P D. Accommodating discontinuities in dimeric left-handed coiled coils in ATP synthase external stalks. Biophys J, 2009, 96: 2823-2831

11 Tiburzy H J, Berzborn R J. Subunit II (b') and not subunit I (b) of photosynthetic ATP synthases is equivalent to subunit $\mathrm{b}$ of the ATP synthases from nonphotosynthetic eubacteria. Evidence for a new assignment of b-type $F_{o}$ subunits. Z Naturforsch C, 1997, 52: 789-798

12 Poetsch A, Berzborn R J, Heberle J, et al. Biophysics and bioinformatics reveal structural differences of the two peripheral stalk subunits in chloroplast ATP synthase. J Biochem, 2007, 141: 411-420

13 Dunn S D, Revington M, Cipriano D J, et al. The b subunit of Escherichia coli ATP synthase. J Bioenerg Biomembr, 2000, 32: 347-355

14 Priya R, Tadwal V S, Roessle M W, et al. Low resolution structure of subunit b (22-156) of Escherichia coli $\mathrm{F}_{1} \mathrm{~F}_{\mathrm{o}}$ ATP synthase in solution and the b-delta assembly. J Bioenerg Biomembr, 2008, 40: 245-255

15 Herrmann R G, Steppuhn J, Herrmann G S, et al. The nuclear-encoded polypeptide $\mathrm{CF}_{\mathrm{o}}$-II from spinach is a real, ninth subunit of chloroplast ATP synthase. FEBS Lett, 1993, 326: 192-198

16 Tiburzy H J, Zimmermann M, Oworah-Nkruma R, et al. Heterologous overexpression of membrane-anchored subunit II of spinach chloroplast ATP synthase and its detergent-free purification as a soluble protein. Z Naturforsch C, 1999, 54: 230-238

17 Sato S, Nakamura Y, Kaneko T, et al. Complete structure of the chloroplast genome of Arabidopsis thaliana. DNA Res, 1999, 6: 283-290

18 Bosco C D, Lezhneva L, Biehl A, et al. Inactivation of the chloroplast ATP synthase gamma subunit results in high non-photochemical fluorescence quenching and altered nuclear gene expression in Arabidopsis thaliana. J Biol Chem, 2004, 279: 1060-1069

19 Maiwald D, Dietzmann A, Jahns P, et al. Knock-out of the genes coding for the Rieske protein and the ATP-synthase delta-subunit of Arabidopsis effects on photosynthesis, thylakoid protein composition, and nuclear chloroplast gene expression. Plant Physiol, 2003, 133: 191-202

20 Inohara $\mathrm{N}$, Iwamoto $\mathrm{A}$, Moriyama $\mathrm{Y}$, et al. Two genes, atpC1 and atpC2, for the gamma subunit of Arabidopsis thaliana chloroplast ATP synthase. J Biol Chem, 1991, 266: 7333-7338

21 Kohzuma K, Bosco C D, Kanazawa A, et al. Thioredoxin-insensitive plastid ATP synthase that performs moonlighting functions. Proc Natl Acad Sci USA, 2012, 109: 3293-3298

22 Qin G, Kang D, Dong Y, et al. Obtaining and analysis of flanking sequences from T-DNA transformants in Arabidopsis. Plant Sci, 2003, 165: 941-949

23 Liu Y G, Mitsukawa N, Oosumi T, et al. Efficient isolation and mapping of Arabidopsis thaliana T-DNA insert junctions by thermal asymmetric interlaced PCR. Plant J, 1995, 8: 457-463

24 Clough S J, Bent A F. Floral dip: A simplified method for agrobacterium-mediated transformation of Arabidopsis thaliana. Plant J, 1998, 16: 735-743

25 Lichtenthaler H K. Chlorophylls and carotenoids: Pigments of pho- 
tosynthetic biomembranes. Methods Enzymol, 1987, 148: 350-382

26 Zhou W, Cheng Y, Yap A, et al. The Arabidopsis gene YS1 encoding a DYW protein is required for editing of $r p o B$ transcripts and the rapid development of chloroplasts during early growth. Plant J, 2009, 58: 82-96

27 Schägger H, Cramer W A, von Jagow G. Analysis of molecular masses and oligomeric states of protein complexes by blue native electrophoresis and isolation of membrane protein complexes by twodimensional native electrophoresis. Anal Biochem, 1994, 217: 220-230

28 Wei J, Xu M, Zhang D, et al. The role of carotenoid isomerase in maintenance of photosynthetic oxygen evolution in rice plant. Acta Biochim Biophys Sin, 2010, 42: 457-463

29 Dong H, Wei J M. Low concentrations of tetracycline enhance the photophosphorylation and $\mathrm{P} / \mathrm{O}$ ratio of chloroplasts. Photosynthesis Res, 2004, 79: 201-208

30 Emanuelsson O, Nielsen H, Brunak S, et al. Predicting subcellular localization of proteins based on their N-terminal amino acid sequence. J Mol Biol, 2000, 300: 1005-1016

31 Small I, Peeters N, Legeai F, et al. Predotar: A tool for rapidly screening proteomes for N-terminal targeting sequences. Proteomics, 2004, 4: 1581-1590

32 Thompson J D, Higgins D G, Gibson T J. CLUSTAL W: Improving the sensitivity of progressive multiple sequence alignment through sequence weighting, position-specific gap penalties and weight matrix choice. Nucleic Acids Res, 1994, 22: 4673-4680

33 Waters M T, Langdale J A. The making of a chloroplast. EMBO J, 2009, 28: 2861-2873

34 López-Juez E, Dillon E, Magyar Z, et al. Distinct light-initiated gene expression and cell cycle programs in the shoot apex and cotyledons of Arabidopsis. Plant Cell, 2008, 20: 947-968

35 Benz M, Bals T, Gügel I L, et al. Alb4 of Arabidopsis promotes assembly and stabilization of a non chlorophyll-binding photosynthetic complex, the $\mathrm{CF}_{1} \mathrm{CF}_{\mathrm{o}}$-ATP synthase. Mol Plant, 2009, 2: 1410-1424

36 Nelson N, Nelson H, Schatz G. Biosynthesis and assembly of the proton-translocating adenosine triphosphatase complex from chloroplasts. Proc Natl Acad Sci USA, 1980, 77: 1361-1364

37 Lill H, Steinemann D, Nelson N. Mutagenesis of the b'-subunit of
Synechocystis sp. PCC 6803 ATP-synthase. Biochim Biophys Acta, 1994, 1184: 284-290

38 Choquet Y, Vallon O. Synthesis, assembly and degradation of thylakoid membrane proteins. Biochimie, 2000, 82: 615-634

39 Stratmann H, Bickel-Sandkötter S. Structure, function, and regulation of chloroplast ATPase. Annu Rev Plant Physiol, 1984, 35: 97-120

40 Lemaire C, Wollman F A. The chloroplast ATP synthase in Chlamydomonas reinhardtii. II. Biochemical studies on its biogenesis using mutants defective in photophosphorylation. J Biol Chem, 1989, 264: $10235-10242$

41 Allen J F. Cyclic, pseudocyclic and noncyclic photophosphorylation: New links in the chain. Trends Plant Sci, 2003, 8: 15-19

42 Abrahams J P, Leslie A G, Lutter R, et al. Structure at 2.8 Å resolution of $F_{1}$-ATPase from bovine heart mitochondria. Nature, 1994, 370: 621-628

43 Löbau S, Weber J, Wilke-Mounts S, et al. $\mathrm{F}_{1}$-ATPase, roles of three catalytic site residues. J Biol Chem, 1997, 272: 3648-3656

44 Motohashi R, Yamazaki T, Myouga F, et al. Chloroplast ribosome release factor 1 (AtcpRF1) is essential for chloroplast development. Plant Mol Biol, 2007, 64: 481-497

45 Xing S, Miao J, Li S, et al. Disruption of the1-deoxy-D-xylulose-5phosphate reductoisomerase (DXR) gene results in albino, dwarf and defects in trichome initiation and stomata closure in Arabidopsis. Cell Res, 2010, 20: 688-700

46 Liu D, Gong Q, Ma Y, et al. cpSecA, a thylakoid protein translocase subunit, is essential for photosynthetic development in Arabidopsis. J Exp Bot, 2010, 61: 1655-1669

47 Oelze-Karow H, Butler W L. The development of photophosphorylation and photosynthesis in greening bean leaves. Plant Physiol, 1971, 48: 621-625

48 Denev I D, Yahubian G T, Minkov I N. Recent advances in chloroplast development in higher plants. In: Pessarakli M, ed. Handbook of Photosynthesis, 2nd ed. New York: Marcel Dekker Press, Inc. 2005. 451-475

49 Baker N R, Butler W L. Development of the primary photochemical apparatus of photosynthesis during greening of etiolated bean leaves. Plant Physiol, 1976, 58: 526-529

Open Access This article is distributed under the terms of the Creative Commons Attribution License which permits any use, distribution, and reproduction in any medium, provided the original author(s) and source are credited.

\section{Supporting Information}

Table S1 Chlorophyll content of wild-type plants and atpg mutants

Table S2 Primers used in northern analysis

Figure S1 Microscopy images of leaves from two-week-old wild type and atpg mutant.

Figure S2 Phenotype of wild type (WT) and complementary plant (atpgc).

The supporting information is available online at csb.scichina.com and www.springerlink.com. The supporting materials are published as submitted, without typesetting or editing. The responsibility for scientific accuracy and content remains entirely with the authors. 